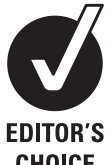

CHOICE

\title{
Sacral nerve stimulation for intractable constipation
}

\author{
Michael A Kamm, ${ }^{1,2}$ Thomas C Dudding, ${ }^{2}$ Jarno Melenhorst, ${ }^{3}$ Michael Jarrett, ${ }^{2}$ \\ Zengri Wang, ${ }^{4}$ Steen Buntzen, ${ }^{5}$ Claes Johansson, ${ }^{6}$ Søren Laurberg ${ }^{5}$ Harald Rosen, ${ }^{7}$ \\ Carolynne J Vaizey, ${ }^{2}$ Klaus Matzel, ${ }^{8}$ Cor Baeten ${ }^{3}$
}

${ }^{1}$ St Vincent's Hospital Melbourne, Australia ${ }^{2}$ St Mark's Hospital, London, UK ${ }^{3}$ Academisch Ziekenhuis, Maastricht, The Netherlands ${ }^{4}$ Medtronic Inc., Minneapolis, Minnesota, USA

${ }^{5}$ Aarhus University Hospital, Aarhus, Denmark

${ }^{6}$ Danderyd Hospital, Danderyd, Sweden

${ }^{7}$ Sozialmedizinisches Zentrum Ost Vienna, Austria

${ }^{8}$ Erlangen University, Germany

\section{Correspondence to}

Professor Michael Kamm, St Vincent's Hospital, University Department of Medicine, Victoria Parade, Fitzroy, Melbourne 3065, Australia; mkamm@unimelb.edu.au

Podium presentation by Dr Thomas Dudding MD MRCS at Digestive Diseases Week, Washington, DC, USA, 19-24 May 2007.

Revised 18 November 2009 Accepted 22 November 2009

\section{ABSTRACT}

Objective Traditional surgical procedures for intractable idiopathic constipation are associated with a variable outcome and substantial morbidity. The symptomatic response, physiological effect and effect on quality of life of sacral nerve stimulation (SNS) were evaluated in patients with constipation (slow transit and normal transit with impaired evacuation).

Methods In a prospective study at five European sites patients who failed conservative treatment underwent 21 days test stimulation. Patients with $>50 \%$ improvement in symptoms underwent permanent neurostimulator implantation. Primary end points were increased defecation frequency, decreased straining and decreased sensation of incomplete evacuation.

Results 62 patients (55 female, median age 40 years) underwent test stimulation, of whom 45 (73\%) proceeded to chronic stimulation. $39(87 \%)$ of these 45 patients achieved treatment success. After a median 28 (range 1-55) months follow-up, defecation frequency increased from 2.3 to 6.6 evacuations per week $(p<0.001)$. Days per week with evacuation increased from 2.3 to $4.8(p<0.001)$. There was a decrease in time spent toileting (10.5 to $5.7 \mathrm{~min}, p=0.001)$, straining (75-46\% of successful evacuations, $p<0.001)$, perception of incomplete evacuation $(71.5-46 \%$ of successful evacuations, $p<0.001)$ and subjective rating of abdominal pain and bloating $(p<0.001)$. Cleveland Clinic constipation score $(0=$ no to $30=$ severe constipation) decreased from 18 to $10(p<0.001)$. Visual analogue scale (VAS) score $(0=$ severe to $100=$ no symptoms) increased from 8 to $66(p<0.001)$. Patients with slow and normal transit benefited. Quality of life significantly improved. Colonic transit normalised in half of those with baseline slow transit $(p=0.014)$.

Conclusion SNS is effective in the treatment of idiopathic slow and normal transit constipation resistant to conservative treatment.

Clinical Trial Number NCT00200005.

\section{INTRODUCTION}

A minority of patients with severe constipation, including some with slow transit and some with normal transit but impaired evacuation, fail conventional pharmacological and behavioural treatments. Traditional operations are associated with substantial morbidity and a variable outcome. ${ }^{1-4}$

Modulation of the extrinsic neural control of the large bowel and pelvic floor may provide an alternative to direct bowel surgery for treating intractable idiopathic constipation. Continuous low-amplitude electrical stimulation of sacral nerve roots is an established treatment for urinary voiding disorders and faecal

\section{What is already known about this subject}

- Many patients with severe idiopathic constipation are resistant to pharmacological and behavioural treatments.

- The traditional surgical treatment for severe constipation is a colectomy with ileorectal anastomosis. This treatment has a variable and unpredictable outcome.

- Sacral nerve stimulation involves low dose chronic neural stimulation via the sacral nerve roots. It is a proven, established therapy for the treatment of faecal incontinence.

\section{What are the new findings}

- Sacral nerve stimulation for treatment of severe idiopathic constipation improves bowel function and relieves the diverse symptoms associated with constipation.

- Symptom improvement is associated with improved gut transit time.

- The benefit from sacral nerve stimulation for severe constipation appears to be maintained in the medium term.

\section{How might it impact on clinical practice in the} foreseeable future

- Sacral nerve stimulation for severe constipation is an effective surgical treatment for patients who have failed conservative treatment.

- This treatment should be considered prior to the consideration of more invasive surgical therapies.

incontinence. ${ }^{5}{ }^{6}$ In a combination of early studies of 250 patients undergoing sacral nerve stimulation (SNS) for urinary voiding disorders, 28 (78\%) of 36 subjects with co-existing symptoms of constipation reported increased frequency of defecation at 6-months follow-up. ${ }^{7-9}$ Other small, preliminary studies, some with limited outcome measures, have reported successful short-term SNS for treating idiopathic constipation. ${ }^{10-13}$

This study aimed to evaluate prospectively the therapeutic efficacy of temporary and permanent SNS in the treatment of idiopathic constipation resistant to medical and behavioural treatment. 


\section{PATIENTS AND METHODS}

A multicentre, prospective, consecutive cohort study was undertaken to evaluate the efficacy of SNS in patients with idiopathic constipation. Patients had a minimum 1 year history of chronic constipation, and failed treatment with laxatives, suppositories, enemas and behavioural therapy (biofeedback). Which drugs had been used, and the nature of the biofeedback, was not specified.

Constipation was defined as two or fewer bowel evacuations per week on average and/or straining to evacuate on $>25 \%$ of attempts to evacuate and/or sensation of incomplete evacuation after defecation on $>25 \%$ of occasions. Although some patients may have also fulfilled criteria for irritable bowel syndrome, this was not formally assessed. Dyssynergia was not assessed and was not part of the entry criteria, as we believe this pattern of muscle function to be an inconsistent and poorly reproducible finding, whose diagnosis differs according to the test being used. ${ }^{14}$

Baseline evaluation included patient completion of a bowel habit diary, subjective questionnaire, visual analogue scale (VAS) score, Cleveland clinic constipation score ${ }^{15}$ and SF-36 (Short-Form 36) quality of life questionnaire. ${ }^{16}$ A physical examination, sigmoidoscopy, anorectal physiological studies, a whole gut transit study ${ }^{17}$ and evacuation proctography ${ }^{18}$ were performed.

The bowel habit diary was completed by the patient over 21 consecutive days, assessing the frequency of attempted and successful defecation, time spent trying to evacuate, presence of straining, need for manual digitation and medications to stimulate defecation, sensation of incomplete evacuation, abdominal pain and bloating, and the impact symptoms had on daily activities of living. During the diary assessment period the patient was asked to abstain from using medications and rectal irrigation. If symptoms became too severe to be tolerated, up to $10 \mathrm{mg}$ of bisacodyl was permitted, with use documented.

A questionnaire completed at the end of the bowel habit diary asked patients to rate subjectively the severity of their constipation, abdominal pain and bloating over the preceding week as absent, mild, moderate or severe. The need to strain to defecate, use of a finger to empty or initiate bowel emptying and feeling of bowel emptiness after defecation during the preceding week were rated as never, sometimes, frequently or always.

Grading of constipation severity was performed using the Cleveland Clinic constipation score that gives a validated, incremental score ranging from 0 , equating to no symptoms, to a maximum of 30 , equating to severe symptoms. ${ }^{15}$ A VAS was also completed asking patients to rate their bowel habit over the previous 3 weeks by placing a mark at an appropriate point along a horizontal line representing very poor bowel habit at one end (minimum score of 0 ) and very good bowel habit at the other (maximum score of 100).

Impact on quality of life was assessed by the SF-36 questionnaire. ${ }^{16}$ This consists of eight domains, each scored from 0 (poor function) to 100 (good function), which assess an individual's physical functioning, physical role, bodily pain, general health, vitality, social functioning, emotional role and mental health.

Anorectal physiological assessment included anal manometry (mean resting and mean incremental squeeze pressures) recorded using a stationary pull-through technique. Rectal sensation to latex balloon distension with air, inflated at a standardised rate of $50 \mathrm{ml} /$ min, was used to measure the rectal sensory threshold, urge threshold and maximal tolerated rectal volume. ${ }^{19}$ Anal and rectal sensitivity to low amplitude electrical stimulation was measured using a catheter-mounted ring electrode placed within the mid-anal canal and upper rectum, respectively. The sensory threshold to electrical stimulation was defined at the first sensation experienced, using stimulation performed at pulse frquency $10 \mathrm{~Hz}$ and pulse width $500 \mu$ s. $^{20}$

Whole gut transit was assessed using three sets of radio-opaque markers of different geometric shapes, with one set given daily over three consecutive days. A plain abdominal radiograph was performed 5 days after the first set of markers was ingested to determine the number of retained markers. Transit was deemed to be slow if an excess of any one of the three sets of markers was retained in comparison with the previously established normal reference range for each set. ${ }^{17}$

Evacuation proctography was performed to assess the anorectal configuration, pelvic floor position and the presence of structural or functional abnormalities before, during and after evacuation of a barium paste enema under fluoroscopic imaging. ${ }^{18}$

For the purpose of analysis, patients were stratified on the basis of their transit time into one of two groups, those who demonstrated slow whole gut transit and those who had normal whole gut transit.

\section{Statistical analysis}

For all measures in this study, each patient served as his or her own control, with baseline data compared with the outcome at last follow-up. Data are presented as mean (SD), median (range) for continuous variables, and count (percentage) for categorical variables. Statistical testing was based on paired t test or Fisher exact test as appropriate, with a significance level of 0.05 . The analysis was by intention to treat.

The study was designed to treat a minimum of 40 patients with permanent implantation. This was based on the number believed to be necessary to provide clear evidence of efficacy, following a result of $>50 \%$ of patients responding in an earlier pilot study.

\section{Exclusion criteria}

Patients were not eligible to enter the study if they had alternating constipation and diarrhoea, congenital or organic bowel pathology, rectal prolapse, previous large bowel surgery, the presence of a stoma or co-existing neurological disease. Those with significant psychological co-morbidity, as assessed subjectively by the investigator, those who were pregnant or those attempting to become pregnant were excluded.

\section{Operative details}

The operative technique for SNS treatment has been described previously and was standardised between centres. ${ }^{21}$ All patients underwent initial percutaneous nerve evaluation (PNE) to establish neural pathway integrity and identify the correct sacral foramen for electrode placement. If a satisfactory response, defined as pelvic floor contraction, was obtained with PNE, then a temporary stimulation wire (Medtronic InterStim model 3057, Minneapolis, Minnesota, USA) was placed and connected to an external pulse generator (Medtronic model 3625). A 3 week screening period of continuous low amplitude stimulation (pulse amplitude $0.1-10 \mathrm{~V} ; 14 \mathrm{~Hz}$; $210 \mu \mathrm{s}$ ) was then commenced during which each subject completed a further bowel habit diary to assess the outcome from test stimulation. At the end of the screening period the temporary wire was removed in all patients.

To be eligible for permanent neurostimulator implantation a patient had to have experienced a subjective improvement of symptoms in the absence of an increase in the use of laxatives, enemas or manual stimulation, as recorded in their bowel habit diary over the 3 week trial period. Minimal criteria for progressing to chronic stimulation were an increase in evacuation frequency to three or more bowel movements per week, and/or a reduction by $\geq 50 \%$ in the number of episodes of straining and/or a decrease by $>50 \%$ in the sensation of incomplete evacuation.

The permanent implantable neurostimulator (INS; Medtronic InterStim Model 3023) was implanted under antibiotic cover in a subcutaneous gluteal pocket, and attached to a tunnelled, quadripolar tined electrode lead (InterStim model 3093/3889) via a short connecting cable (InterStim model 3095). 
Initial stimulation parameters for permanent stimulation were set normally at $14 \mathrm{~Hz}$ (range $10-21 \mathrm{~Hz}$ ), $210 \mu$ s, continuous stimulation with the amplitude of stimulation set at just below the patient's sensory threshold. ${ }^{22}$ A desirable electrode configuration was achieved when the patient experienced sensation localised near, or within, the anus with stimulation amplitude set at the sensory threshold.

\section{Assessment and follow-up}

Patients were reviewed, with bowel habit diary assessment, symptom questionnaires, VAS score, Cleveland Clinic constipation score and SF-36 quality of life questionnaire completed at 1, 3, 6 and 12 months following implantation of the permanent device, and at yearly intervals thereafter. The diary card evaluations were all undertaken with the patient not using laxatives. Anorectal physiological studies were performed at each follow-up. A whole gut transit study and evacuation proctography were repeated at 6 months following permanent implantation.

Undesirable symptoms occurring during the study were documented as an adverse event, regardless of whether they were considered to be related to the treatment. The severity of these events was classified as mild, moderate or severe, using standard international criteria.

The primary outcome measure of treatment success was defined in each patient as improvement in any one of: (1) bowel frequency changing from two or less to three or more evacuations per week; (2) $a \geq 50 \%$ reduction in the proportion of defecation episodes associated with straining; or (3) a $\geq 50 \%$ reduction in the proportion of defecation episodes associated with a sense of incomplete evacuation. The use of improvement of any one of the three main symptoms was designed to reflect the spectrum of symptoms that patients with this condition complain of.

Assessment was also made of the number of patients who had improved all their abnormal inclusion criteria at the end of followup, each subject acting as their own control.

The trial was performed in accordance with the 1975 Declaration of Helsinki. Ethical approval from each institution participating in the study was obtained and every patient provided written, informed consent.

\section{RESULTS}

Sixty-two patients, 55 (89\%) female, with a median age of 40 (range 17-79) years were enrolled in the study. Thirty patients (48\%) were recruited from St Mark's Hospital, London, UK; 17 patients (27\%) from Academisch Ziekenhuis Maastricht, The Netherlands; eight patients (13\%) from Aarhus University Hospital, Denmark; four patients (7\%) from Danderyd University Hospital, Sweden; and three patients (5\%) from Sozialmedizinisches Zentrum Ost, Vienna, Austria.

All had idiopathic constipation that was refractory to maximal medical and behavioural treatment. Symptoms of constipation had been present for a median duration of 10 (range 1-60) years prior to study enrolment.

Fifty patients (81\%) demonstrated slow colonic transit (group 1) and 12 patients (19\%) normal colonic transit (group 2). There was no significant difference in baseline demographics or severity of symptoms between the two groups of patients.

All patients completed PNE followed by insertion of a temporary stimulation electrode. Screening in all patients was performed for a median of 21 (range 1-38) days. Six patients underwent a repeat test procedure and screening evaluation due to lead damage or loss of efficacy secondary to dislocation of the temporary stimulation electrode. Three of these six subjects met the criteria for permanent implantation. A total of 45 of the 62 patients (73\%) met one or more criteria to proceed to implantation of a permanent device. All 45 patients who met the implant criteria proceeded to implantation of a permanent electrode lead and INS. Of these patients, 37 (82\%) had slow transit constipation and eight (18\%) normal transit constipation with impaired evacuation.

The foramen for permanent lead implantation was based on the best motor response during acute operative nerve testing, being S3 in $41(91 \%)$ patients, S2 in one (2\%) patient and S4 in three $(7 \%)$ patients. Median (range) initial stimulation parameter settings were: pulse amplitude $1.25(0.3-4.0) \mathrm{V}$, pulse frequency $14 \mathrm{~Hz}^{10-21}$ and pulse width $210 \mu \mathrm{s}$.

Results of chronic SNS are reported at latest follow-up, median 28 (range 1-55) months following permanent implantation.

\section{Clinical outcome}

Thirty-nine (87\%) of the 45 permanently implanted patients were classified as having achieved treatment success, meeting at least one primary end point, based on their inclusion criteria, at latest followup. Fifteen of the 45 patients (33\%) improved all their abnormal inclusion criteria at latest follow-up. The number of patients enrolled by different inclusion criteria and their matched subsequent outcome from permanent SNS is illustrated in figure 1. On an intention-to-treat basis, 39 of all 62 patients (63\%) enrolled in the study met the primary end point definition of a successful treatment outcome at latest follow-up.

The results of chronic stimulation at each stage of follow-up are summarised in table 1 . There was no significant difference in the success rate of temporary or permanent stimulation between centres.

There was a significant increase in frequency of defecation from a median (range, mean) of $2.3(0-20,3.6)$ evacuations per week at baseline to $6.6(1-16,6.6)$ evacuations per week at most recent follow-up $(p<0.001)$. Spontaneous bowel movements-that is, those occurring without laxatives or other stimulation-increased from a median (range, mean) of $1.7(0-14,2.5)$ per week at baseline to 4.3 $(0-12,4.6)$ at latest follow-up $(p=0.004)$. Defecation was significantly more likely to be associated with successful evacuation after permanent SNS, compared with baseline (figure $2 ; \mathrm{p}=0.018$ ).

The number of days per week with successful defecation increased significantly $(p<0.001)$ and is shown in figure 3 . The time spent on toileting decreased significantly $(p=0.001)$ and is shown in figure 4 .

A reduction in percentage of episodes during which straining was required to open the bowels was seen with SNS. Straining was present for $75 \%$ of all successful evacuations at baseline versus $46 \%$ of all successful evacuations at latest follow-up $(p<0.001)$. There was a significant reduction in the percentage of successful evacuations associated with a sensation of incomplete evacuation, from $71 \%$ at baseline to $46 \%$ at latest follow-up $(\mathrm{p}<0.001)$.

There was an improvement in the symptoms associated with constipation. The number of days per week that abdominal pain was experienced decreased from a median (range, mean) of 5 $(0-7,4.5)$ at baseline to $1.7(0-7,2.3)$ days per week at latest followup $(\mathrm{p}<0.001)$. The number of days abdominal bloating was experienced decreased from a median of $5.7(0-7,4.7)$ to $2.3(0-7,2.9$; $p<0.001)$. On subjective rating of the overall severity of abdominal pain and bloating as absent, mild, moderate or severe, there was a significant improvement in both symptoms with chronic stimulation (figure 5).

The Cleveland Clinic constipation score $(0=$ no symptoms of constipation to $30=$ severe constipation) decreased significantly $(p<0.001)$ and is shown in figure 6. Grading of the severity of symptoms by $\operatorname{VAS}(0=$ poor function to $100=$ best function) demonstrated a subjective improvement in constipation with chronic stimulation, with the score increasing from a median (range) of $8(0-100,15)$ to $66(11-100,63 ; p<0.001$; figure 7$)$. Medication usage was documented and was found to be constant at each stage of follow-up ( $p=0.753)$. 
Greater than $50 \%$ reduction in symptoms at latest follow up / Evacuation frequency of $>3$ per week at latest follow up

$25-50 \%$ reduction in symptoms at latest follow-up

$\square$ Less than $25 \%$ reduction in symptoms at latest follow up I Evacuation frequency of two or less per week at latest follow-up

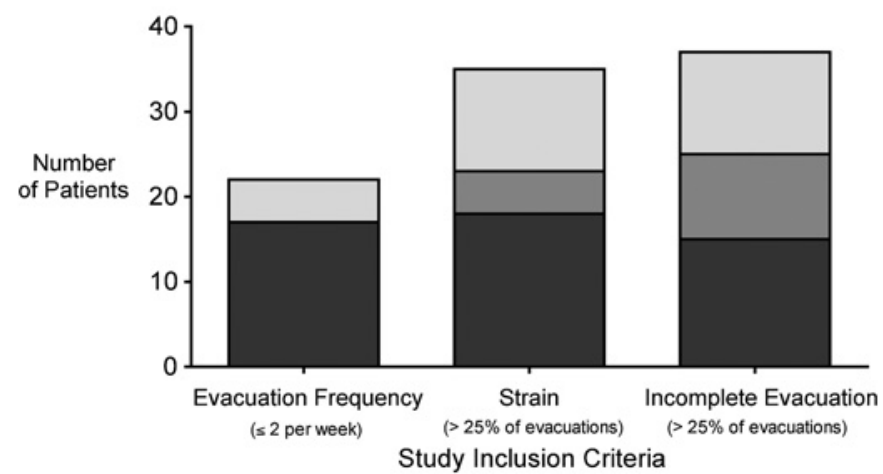

Figure 1 Number of patients enrolled by different inclusion criteria (evacuation frequency, straining $>25 \%$ of all evacuations, sensation of incomplete evacuation $>25 \%$ of all evacuations) and matched subsequent outcome for individuals with that criterion after chronic sacral nerve stimulation at a median of 28 (range $1-55$ ) months follow-up.

Patients with both slow and normal transit achieved significant treatment success with improved defecation frequency, reduction in straining and improvement in other symptoms.

\section{Quality of life}

There was significant improvement in four of the eight subsets measured by the SF-36 questionnaire. Bodily pain (median 37 at baseline vs 49 at latest follow-up; $p=0.001$ ), mental health (39 vs 46; $\mathrm{p}=0.027$ ), social functioning ( 40 vs $51 ; \mathrm{p}=0.008$ ) and vitality (median 36 vs 46; 0.003 ) were significantly improved, while general health, physical functioning, emotional and physical role scores did not change significantly (figure 8)

\section{Physiological data}

There was no significant change when comparing anal manometric findings performed at baseline and after 6 months chronic stimulation.

Sensory function changed with treatment. The sensory threshold to rectal balloon distension decreased by a non-significant degree (median $30 \mathrm{ml}$ at baseline vs $29 \mathrm{ml}$ at 6 months, mean $48 \mathrm{vs} 34 \mathrm{ml}$, $\mathrm{p}=0.09)$. There was a significant reduction in the urge threshold (median $76 \mathrm{ml}$ at baseline vs $74 \mathrm{ml}$ at 6 months, mean 95 vs $72 \mathrm{ml}$, $\mathrm{p}=0.007$ ) and maximal tolerated threshold (median $138 \mathrm{ml}$ at baseline vs $103 \mathrm{ml}$ at 6 months, mean $151 \mathrm{vs} 107 \mathrm{ml}, \mathrm{p}<0.001$ ) to rectal balloon distension with chronic stimulation.

\section{Whole gut transit}

Paired whole gut transit data were available in 27 patients. Of these, $20(74 \%)$ had delayed whole gut transit at baseline compared with 9 $(33 \%)$ at 6 months of permanent chronic stimulation $(p=0.014)$. In those subjects who normalised their whole gut transit time with SNS, frequency of defecation increased from a median (range) of 2.7 (0.5-8.7) at baseline to $6.5(3.2-12.7)$ evacuations per week at 6 months $(p=0.008)$. In those in whom no improvement in transit was observed, there was no significant change in the frequency of evacuation, from a median (range) of $3.2(1.4-11.2)$ at baseline to 4.6 $(2.5-8.3)$ at 6 months $(p=0.456)$.

\section{Proctogram}

Paired proctogram data were available in 22 patients. Of these, 12 patients $(55 \%)$ had prolonged evacuation at baseline compared with $7(32 \%)$ at 6 months of chronic SNS $(p=0.642)$. Six patients had complete evacuation at baseline (27\%), improving to 13 patients $(59 \%)$ at 6 months follow-up ( $\mathrm{p}=0.046)$

\section{Adverse events}

One hundred and one adverse events were reported, of which 40 $(40 \%)$ were attributed to underlying constipation or a new unrelated diagnosis.

Of the adverse events that were related to the treatment, over two-thirds were classified as mild, these being mainly secondary to postoperative discomfort that resolved spontaneously or adverse

Table 1 Effects of chronic sacral nerve stimulation on the symptoms of constipation compared with prestimulation baseline severity

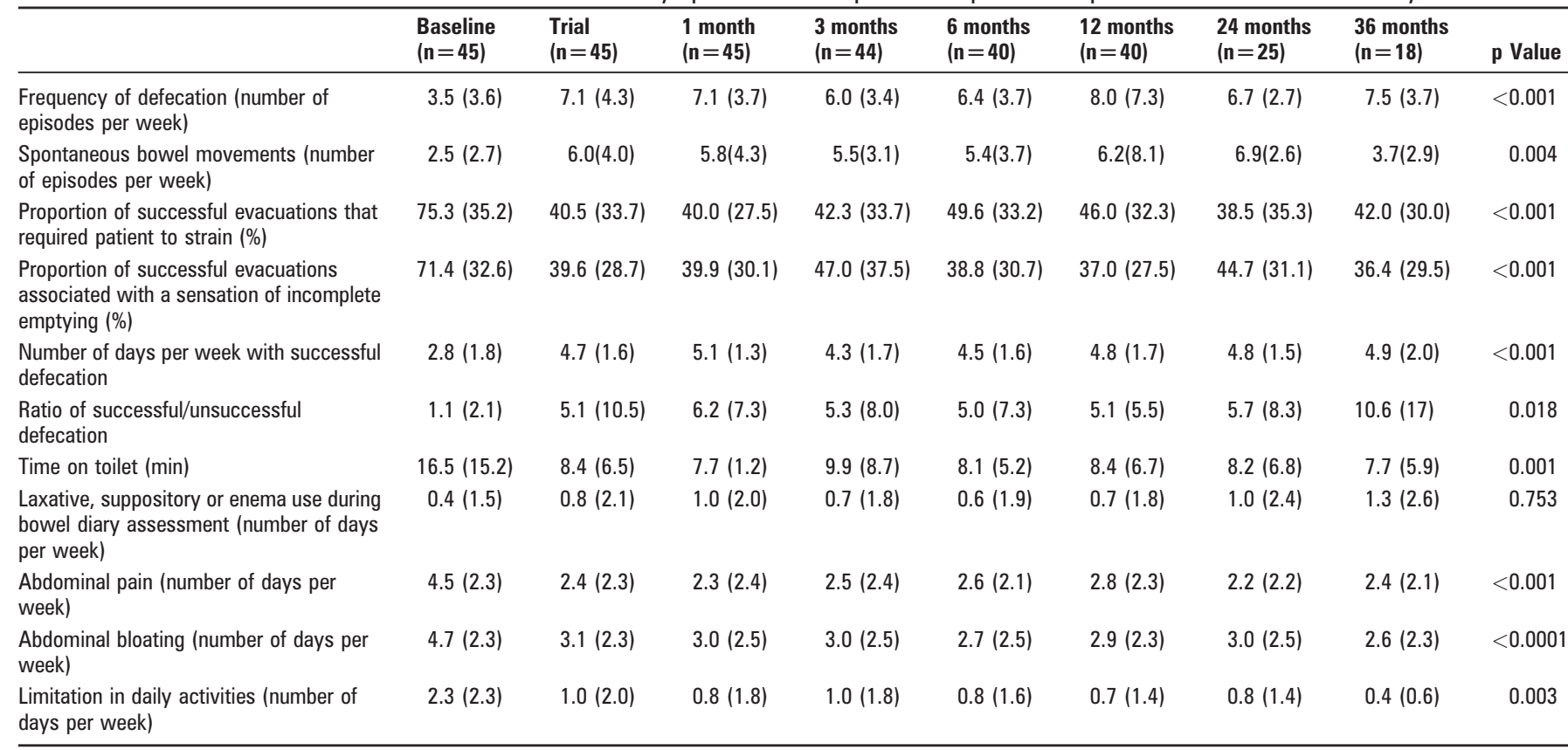

Values are expressed as mean (SD) at baseline and at each stage of follow-up. 


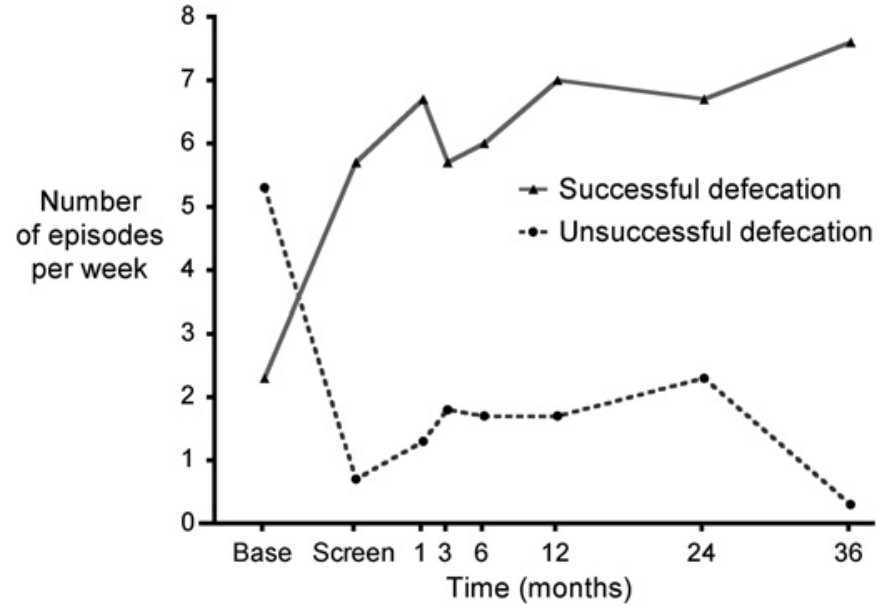

Figure 2 Median number of episodes of successful and unsuccessful evacuation in patients attempting to defecate, recorded by a 3 week bowel habit diary before and after chronic sacral nerve stimulation $(p=0.018)$.

stimulation that was eliminated following re-programming of the INS

There were 11 severe adverse events related to treatment. Two patients developed a deep postoperative infection necessitating removal of the INS; in one of these patients a further device was inserted once the infection had been treated. One patient required further surgery to remove and replace a stimulation lead that had eroded superficially through the skin. Two patients experienced persistent postoperative pain at the site of INS implantation that necessitated moving the INS to a new implant site. Four patients underwent elective lead revision, three for adverse stimulation that was persistent despite re-programming of the INS and one for suspected lead migration. Two patients experienced device failure that required further surgery to replace the defective component.

Women who were pregnant, or considering getting pregnant, were excluded from study entry. One patient, however, had two pregnancies during the course of the study. In the first pregnancy, stimulation was ceased at 9 weeks gestation and the patient had a subsequent premature delivery at 29 weeks to an infant with Down syndrome. In the second pregnancy, stimulation was stopped early in the first trimester and a healthy baby was born at 38 weeks by elective caesarean section. The patient successfully resumed treatment after these pregnancies.

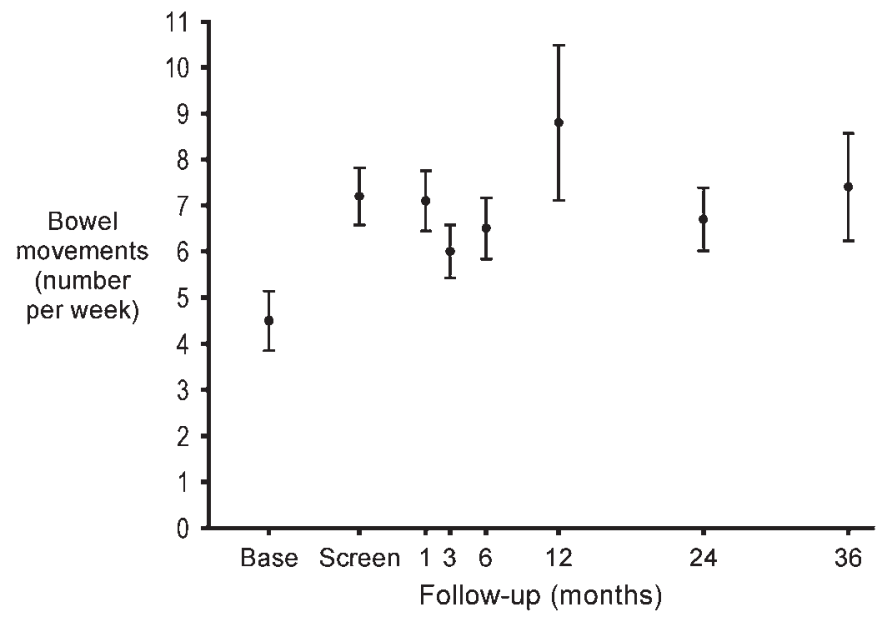

Figure 3 Mean (SD) number of evacuations per week in patients, recorded by a 3 week bowel habit diary before and after chronic sacral nerve stimulation.

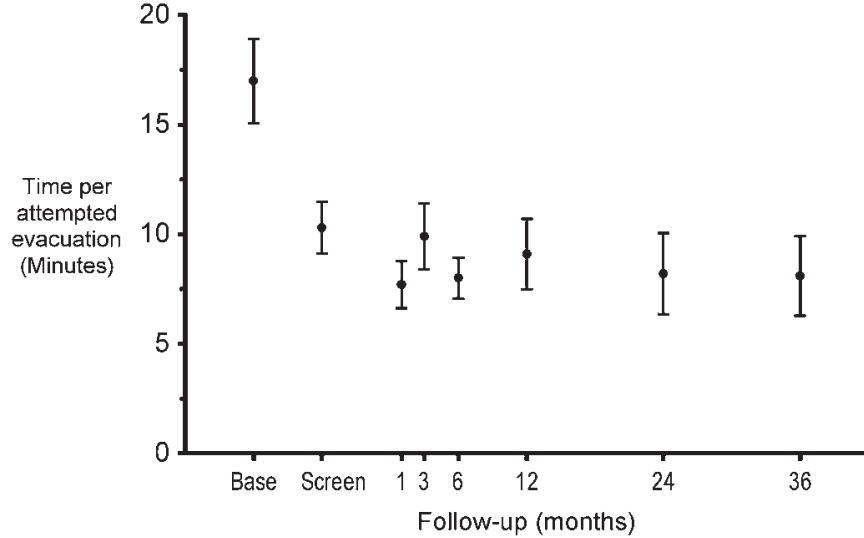

Figure 4 Mean (SD) duration of time spent on toileting for each attempted bowel evacuation, recorded by bowel habit diary before and after chronic sacral nerve stimulation.

Seven patients exited from the study (figure 9). Three patients expressed a wish not to continue participation in the study, two patients exited due to lack of efficacy, one patient declined a further INS after their infected implant was removed and one patient underwent surgery in the form of a subtotal colectomy.

\section{DISCUSSION}

This study has demonstrated that SNS is an effective treatment for intractable idiopathic constipation in patients who have failed to respond to maximal conservative treatments. The effect of stimulation on bowel function is rapid, with a significant improvement in

a Abdominal Pain

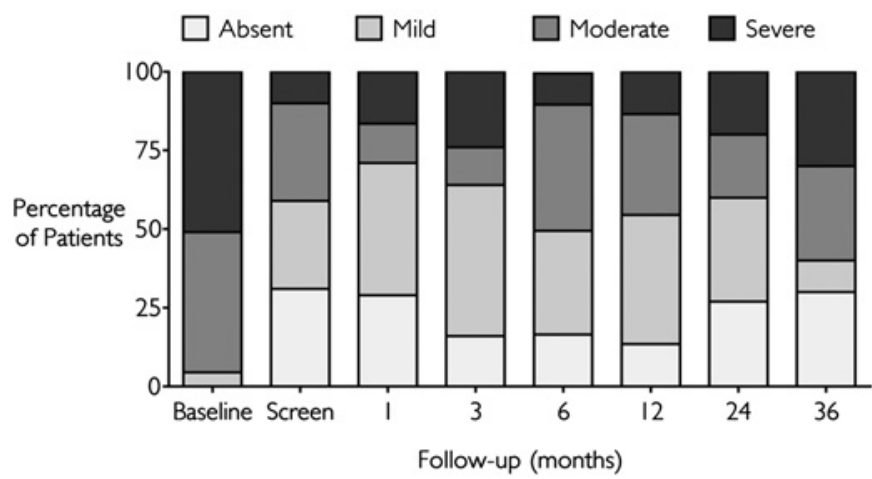

b

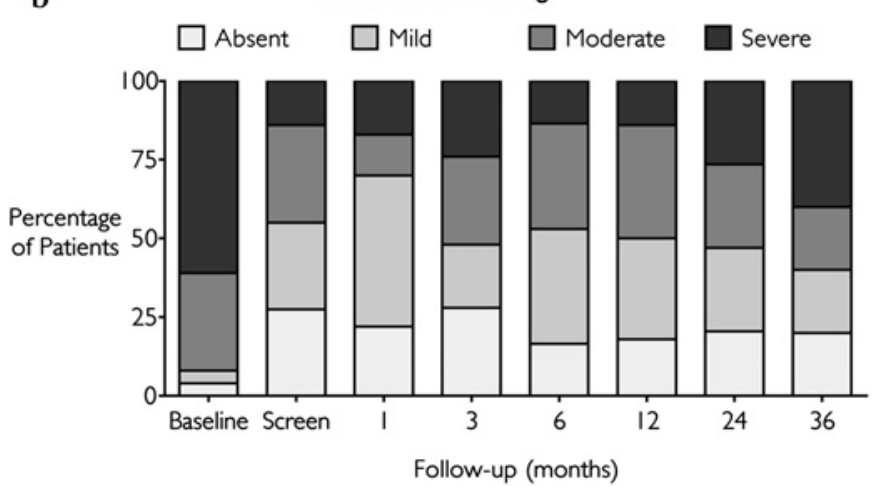

Figure 5 Subjective rating of (a) abdominal pain and (b) abdominal bloating at baseline and with chronic sacral nerve stimulation, as recorded by symptom questionnaire. Patients rated each of these symptoms as absent, mild, moderate or severe. 


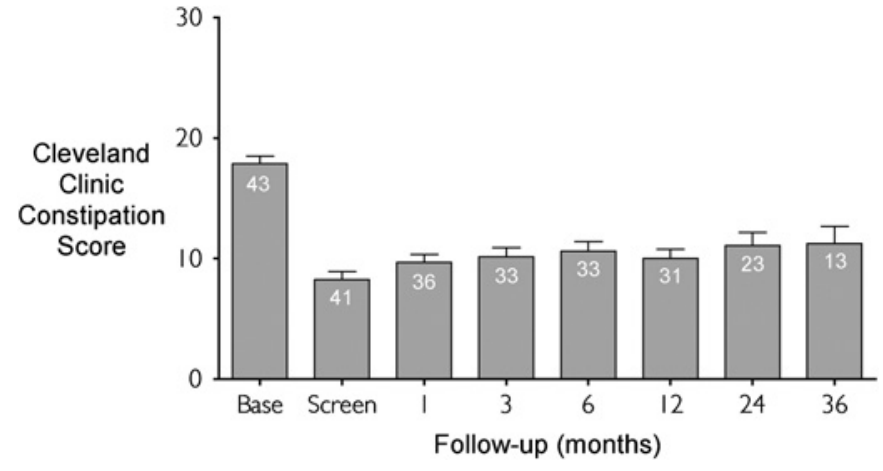

Figure 6 Mean (SD) Cleveland Clinic constipation score before and after chronic sacral nerve stimulation. The mean number of patients at each stage of follow-up is shown within the bars.

symptoms occurring with temporary screening. This effect is maintained in the medium to long term, with improvement in a range of outcome measures.

This was intended to be a real-life study, to include patients with a spectrum of symptoms. However, patients were classified prospectively into those with normal and slow transit, to gain some degree of homogeneity of groups. All patients had failed full medical treatment. Patients had severe enough symptoms subjectively to pursue this intervention; the Cleveland Clinic scores were high, suggesting severe subjective symptoms.

This study suggests that SNS is effective for severe idiopathic constipation

The definition used for constipation in this study differs from that of some other pharmacological studies; direct comparisons should therefore be undertaken with caution. There are no universally accepted standardised inclusion criteria for patients undergoing clinical trials for constipation.

The Rome criteria require two or more predefined symptoms to be present for a minimum of 3 months. In this study, patients had to exhibit one of three symptoms to be eligible for test stimulation. Thirtyfour of the 45 patients undergoing permanent stimulation had two or more inclusion criteria and would have satisfied the Rome criteria.

Patients were deemed as having a successful response to treatment if one or more of the symptoms for which they were included in the trial significantly improved. This definition may make comparison of the results with other studies difficult to interpret.

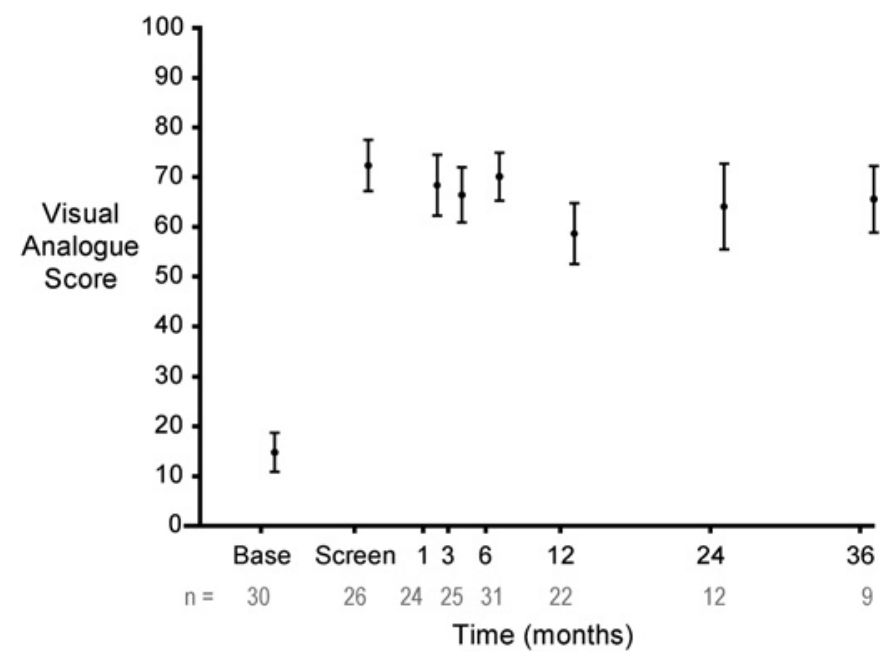

Figure 7 Subjective grading of the severity of constipation in those patients undergoing sacral nerve stimulation as measured by mean (SD) visual analogue score $(p<0.001)$. Score ranging from $0=$ worst function to $100=$ best function.

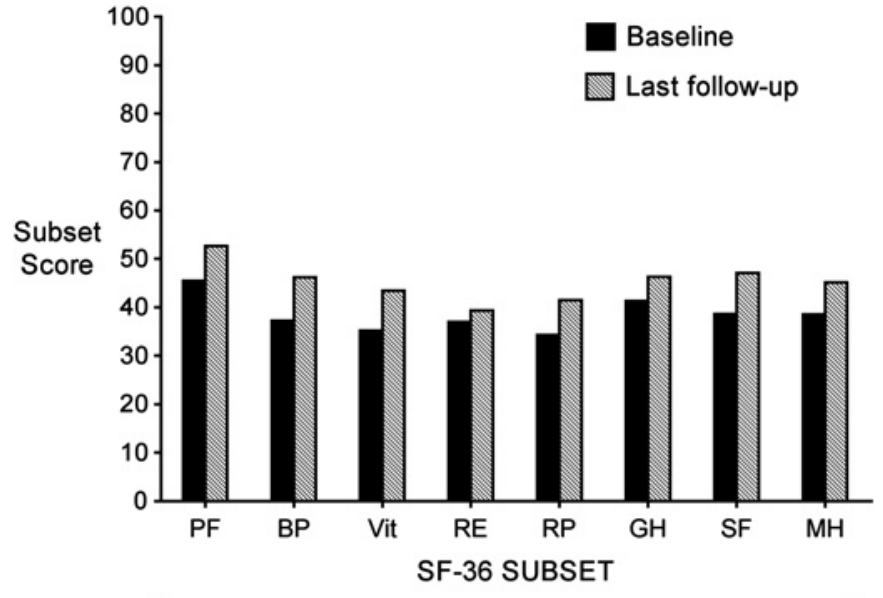

PF - physical function; BP - bodily pain; Vit - vitality; RE - role emotiona $\mathrm{RP}$ - role physical; GH - general health; SF - social functioning; MH - mental health

Figure 8 Mean Short-Form 36 (SF-36) subset scores at baseline and following chronic sacral nerve stimulation at latest follow-up.

Life expectancy of the battery is 4-7 years, depending on stimulation parameters and the device used. The battery can then be changed operatively.

This was designed to be a real-life long-term evaluation in a prospective consecutive series of patients for up to 5 years, with a minimum of 1 year duration. To our knowledge no previous controlled study of treatment for idiopathic constipation has been conducted over the length of time that the current study was conducted; it is therefore difficult to estimate what might be a placebo response over such a long time period. However, we believe that the therapeutic benefit demonstrated over this long time course is very unlikely to relate to a placebo effect. Furthermore, in addition to subjective improvement, there was an objective improvement in transit time and evacuation time on proctography. A previous small, double-blind, cross-over study has demonstrated that the beneficial effects of this treatment are unlikely to relate to a placebo effect. ${ }^{23}$

We do not have data on the exact quantity of laxatives used during follow-up, although laxative use was only a mean of 1 day per week at last follow-up.

Quality of life improved significantly in six of the eight SF-36 domains. Comparison was not made with population norms, as they were not available for all the countries in which this study took place; however, quality of life generally did not improve to the normal level of the US population (data not shown). The SF-36 is not "disease specific" and can be influenced by co-existing illness that was not relieved by SNS.

Patients with both slow and normal transit benefited from SNS. Treatment resulted in improvement of all symptoms and objective improvement in transit time and evacuation time on proctography. Slow transit and impaired evacuation often overlap. ${ }^{14}$ Studies in healthy volunteers have shown that the suppression of defecation by pelvic floor contraction can result in the retrograde movement of colonic contents and delayed transit. ${ }^{24}$ In contrast, colonic transit can be normalised following pelvic floor-focused behavioural therapy ("biofeedback"). ${ }^{25}$

At baseline the median frequency of defecation was greater than three evacuations per week, with 22 patients reporting a bowel frequency of less than twice per week. A number of patients had multiple attempts to open their bowels within a day, passing small amounts on each occasion but never completely evacuating. This was reflected in the low mean number of days of successful evacuation. Alternative inclusion criteria included an excessive proportion of evacuations during which the patient strained, or an excessive proportion of evacuations in which the patient felt symptoms of incomplete emptying. These subjective abnormalities 
Figure 9 Flow diagram to show the passage of participants through each stage of the trial. SNS, sacral nerve stimulation.

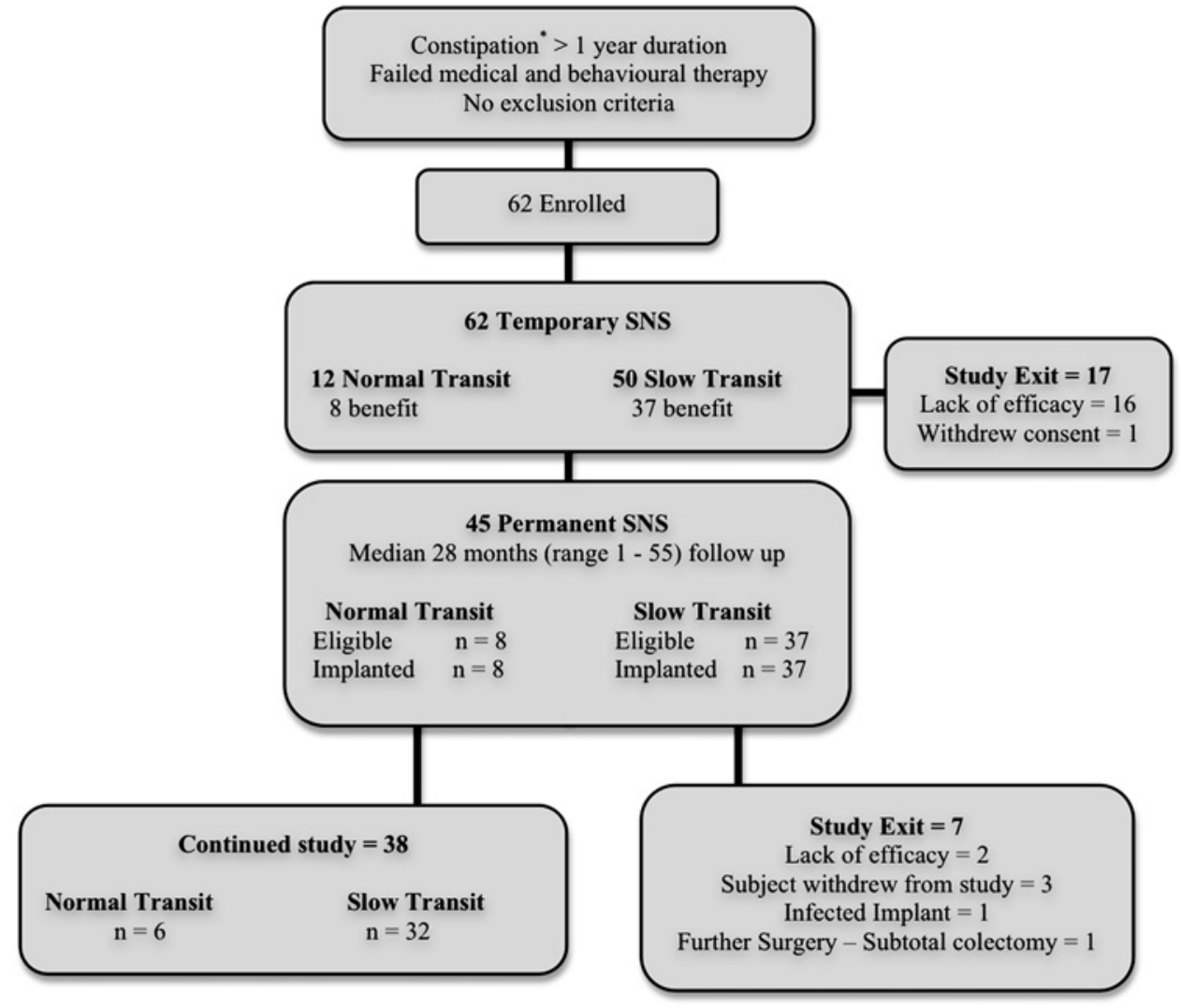

* = Constipation Defined as:

Two or fewer bowel evacuations per week on average

and/or straining to evacuate on over $25 \%$ of attempts to evacuate

and/or sensation of incomplete evacuation after defecation on over $25 \%$ of occasions

were associated with objectively measured slow transit in the majority $(80 \%)$ of patients.

Some patients failed to benefit from temporary SNS. This may relate to neuromuscular pathology or psychological morbidity. There are no techniques available to indicate the former reliably. Misplacement or migration of the temporary electrode can occur and account for failure.

One in eight patients (13\%) who responded to temporary screening failed to benefit from chronic stimulation. This may relate to a placebo effect during temporary screening, a positive early effect diminishing over several weeks, surgical misplacement of the quadripolar electrode lead or late lead dislocation. ${ }^{26}$ Inaccurate patient screening diaries, unreported medication use, changes in stool consistency or persistent undesirable learned behaviour such as straining may be alternative factors. Further studies on a range of stimulation parameters may benefit patients with an incomplete or absent response. ${ }^{27}$

Understanding of the precise mechanism of action of SNS for constipation remains incomplete. A number of different neural pathways may be involved. Stimulation is performed at a low level and continuously, in contrast to the acute intermittent high level stimulation used with the Brindley stimulator in spinal cord-injured patients. ${ }^{28}$ Effects are seen on motor, ${ }^{29}$ sensory ${ }^{12}$ and central neural pathways. ${ }^{30}$ The effect is therefore not a straightforward result of acute increased peristaltic motor activity through activation of efferent nerves.

In this study transit time was seen to normalise in some patients undergoing chronic stimulation. An increase in the frequency of pancolonic antegrade propagating sequences following high amplitude stimulation of the third sacral nerve root has previously been demonstrated. ${ }^{29}$ Measured sensory function within the rectum also appeared to be affected by chronic SNS. This may reflect altered perception of rectal content, or may just be a surrogate marker of altered autonomic activity.

The incidence of adverse events was similar to that of previous studies. ${ }^{56}$ This procedure has low morbidity and is well tolerated, in marked contrast to major resectional bowel surgery. Whether the birth of an infant with Down syndrome was related to the SNS is unknown. In one previous report of six pregnancies occurring in patients undergoing SNS for urological disorders the only adverse outcome was a premature delivery in one patient. ${ }^{31}$

In conclusion, SNS is an effective treatment for patients with intractable constipation unresponsive to conservative treatments. Benefit is maintained, at least in the medium term. Further randomised trial data are now awaited.

Funding This study was sponsored and monitored by Medtronic Europe Sarl. The study design, analysis and interpretation of data and subsequent writing of this report were performed without the influence of Medtronic.

Competing interests Declared (the declaration can be viewed on the Gut website at http://www.gut.bmj.com/supplemental)

Ethics approval This study was conducted with the approval of the individual ethics committees at each research site.

Patient consent Obtained

Provenance and peer review Not commissioned; externally peer reviewed.

\section{REFERENCES}

1. Kamm MA, Hawley PR, Lennard-Jones JE. Outcome of colectomy for severe idiopathic constipation. Gut 1988:29:969-73.

2. Wong SW, Lubowski DZ. Slow-transit constipation: evaluation and treatment. ANZ J Surg 2007;77:320-8.

3. Lundin E, Karlbom U, Pahlman L, et al. Outcome of segmental colonic resection for slow-transit constipation. Br J Surg 2002;89:1270-4. 
4. Lees NP, Hodson P, Hill J, et al. Long-term results of the antegrade continent enema procedure for constipation in adults. Colorectal Dis 2004;6:362-8.

5. Brazzelli M, Murray A, Fraser C. Efficacy and safety of sacral nerve stimulation for urinary urge incontinence: a systematic review. J Urol 2006;175:835-41.

6. Mowatt G, Glazener C, Jarrett M. Sacral nerve stimulation for faecal incontinence and constipation in adults. Cochrane Database Syst Rev 2007;(3): CD004464

7. Hassouna MM, Siegel SW, Nyeholt AA, et al. Sacral neuromodulation in the treatment of urgency-frequency symptoms: a multicenter study on efficacy and safety. J Urol 2000;163:1849-54.

8. Jonas U, Fowler CJ, Chancellor MB, et al. Efficacy of sacral nerve stimulation for urinary retention: results 18 months after implantation. J Urol 2001;165:15-19.

9. Schmidt RA, Jonas U, Oleson KA, et al. Sacral nerve stimulation for treatment of refractory urinary urge incontinence. Sacral Nerve Stimulation Study Group. J Urol 1999;162:352-7.

10. Masin A, Ratto C, Ganio E, et al. Effect of sacral nerve modulation in chronic constipation. In: ECCP/EACP second joint meeting. Bologna, Italy: Ital J Publi Health 2005;2:305

11. Malouf AJ, Wiesel PH, Nicholls T, et al. Short-term effects of sacral nerve stimulation for idiopathic slow transit constipation. World J Surg 2002:26:166-70.

12. Kenefick NJ, Nicholls RJ, Cohen RG, et al. Permanent sacral nerve stimulation for treatment of idiopathic constipation. Br J Surg 2002;89:882-8.

13. Holzer B, Rosen HR, Novi G, et al. Sacral nerve stimulation in patients with severe constipation. Dis Colon Rectum 2008;51:524-29; discussion 529-30.

14. Kamm MA. Clinical case: chronic constipation. Gastroenterology 2006;131:233-9.

15. Agachan F, Chen T, Pfeifer J, et al. A constipation scoring system to simplify evaluation and management of constipated patients. Dis Colon Rectum 1996;39:681-5

16. Jenkinson C, Coulter A, Wright L. Short form 36 (SF36) health survey questionnaire: normative data for adults of working age. BMJ 1993;306:1437-40.

17. Evans RC, Kamm MA, Hinton JM, et al. The normal range and a simple diagram for recording whole gut transit time. Int J Colorectal Dis 1992:7:15-17.
18. Mahieu P, Pringot J, Bodart P. Defecography: I. Description of a new procedure and results in normal patients. Gastrointest Radiol 1984;9:247-51.

19. Sun WM, Read NW, Prior A, et al. Sensory and motor responses to rectal distention vary according to rate and pattern of balloon inflation. Gastroenterology 1990:99:1008-15.

20. Kamm MA, Lennard-Jones JE. Rectal mucosal electrosensory testing-evidence fo a rectal sensory neuropathy in idiopathic constipation. Dis Colon Rectum 1990:33:419-23

21. Spinelli M, Giardiello G, Arduini A, et al. New percutaneous technique of sacral nerve stimulation has high initial success rate: preliminary results. Eur Urol 2003:43:70-4.

22. Koch SM, van Gemert WG, Baeten CG. Determination of therapeutic threshold in sacral nerve modulation for faecal incontinence. Br J Surg 2005:92:83-7.

23. Kenefick NJ, Vaizey CJ, Cohen CR, et al. Double-blind placebo-controlled crossover study of sacral nerve stimulation for idiopathic constipation. Br J Surg 2002;89:1570-1.

24. Halls J. Bowel content shift during normal defaecation. Proc $R$ Soc Med 1965;58:859-60.

25. Emmanuel AV, Kamm MA. Response to a behavioural treatment, biofeedback, in constipated patients is associated with improved gut transit and autonomic innervation. Gut 2001;49:214-9.

26. Deng DY, Gulati $\mathrm{M}$, Rutman $\mathrm{M}$, et al. Failure of sacral nerve stimulation due to migration of tined lead. J Urol 2006;175:2182-5.

27. Dudding TC, Vaizey CJ, Gibbs A, et al. Improving the efficacy of sacral nerve stimulation for faecal incontinence by alteration of stimulation parameters. $\mathrm{Br} J$ Surg 2009;96:778-84

28. Brindley GS, Rushton DN. Long-term follow-up of patients with sacral anterior root stimulator implants. Paraplegia 1990;28:469-75.

29. Dinning PG, Fuentealba SE, Kennedy ML, et al. Sacral nerve stimulation induces pancolonic propagating pressure waves and increases defecation frequency in patients with slow-transit constipation. Colorectal Dis 2007;9:123-32.

30. Sheldon R, Kiff ES, Clarke A, et al. Cortical reorganization induced by sacral nerve stimulation in patients with faecal incontinence. Colorectal Dis 2004;6:23.

31. Wiseman 0J, v d Hombergh U, Koldewijn EL. Sacral neuromodulation and pregnancy. J Urol 2002:167:165-8.

\section{Gut tutorial}

\section{Management of Crohn's disease}

This is an introduction to the Gut tutorial 'Management of Crohn's disease' hosted on BMJ Learning - the best available learning website for medical professionals from the BMJ Group.

This interactive module is focused around a case of Crohn's disease and its medical and surgical management over a period of 15 years from adolescence to adulthood. The module is designed to focus the reader's consideration on the key current and historic evidence base that has shaped modern management of the condition both in adults and children. The target audience is gastroenterology trainees, gastroenterology nurse specialists and consultants in gastroenterology treating patients with inflammatory bowel disease. Specific areas covered by the module include diagnostic pathology and radiology; medical management including nutritional therapy, corticosteroids, immunomodulators and biological therapy; as well as surgical management, lifestyle modification and psychosocial considerations.

The evidence base cited in this module incorporates research published until November 2009 .

To access the tutorial (Interactive Case History), click on BMJ Learning: Take this module on BMJ Learning from the content box at the top right and bottom left of the online article. For more information please go to: http://gut.bmj.com/site/tutorials/

If prompted, subscribers must sign into Gut with their journal username and password. All users must also complete a one-time registration on BMJ Learning and subsequently log in (with a BMJ Learning username and password) on every visit.

\section{CA Lamb, ${ }^{1}$ RA Speight, ${ }^{1}$ S Needham, ${ }^{2}$ JY Graham, ${ }^{3}$ JC Mansfield ${ }^{1}$}

${ }^{1}$ Gastroenterology Department, Newcastle upon Tyne Hospitals NHS Foundation Trust, Royal Victoria Infirmary, Newcastle upon Tyne, UK; ${ }^{2}$ Histopathology Department, Newcastle upon Tyne Hospitals NHS Foundation Trust, Royal Victoria Infirmary, Newcastle upon Tyne, UK; ${ }^{3}$ Radiology Department, Newcastle upon Tyne Hospitals NHS Foundation Trust, Royal Victoria Infirmary, Newcastle upon Tyne, UK

Correspondence to Dr John C. Mansfield, Gastroenterology, Newcastle upon Tyne Hospitals NHS Foundation Trust, Royal Victoria Infirmary, Queen Victoria Road, Newcastle upon Tyne NE1 4LP, UK; John.Mansfield@nuth.nhs.uk

\section{Competing interests None.}

Patient consent Obtained.

Provenance and peer review Commissioned; not externally peer reviewed. 